\title{
Utilidad de la Prueba de Micronúcleos y Anormalidades Nucleares en Células Exfoliadas de Mucosa Oral en la Evaluación de Daño Genotóxico y Citotóxico
}

\author{
Utility Micronucleus Test and Nuclear Abnormalities in Exfoliated Cells \\ of Oral Mucosa in the Evaluation of Genotoxic and Cytotoxic Damage
}

Olivia Torres-Bugarín* \& María Luisa Ramos-Ibarra**

TORRES-BUGARÍN, O. \& RAMOS-IBARRA, M. L. Utilidad de la prueba de micronúcleos y anormalidades nucleares en células exfoliadas de mucosa oral en la evaluación de daño genotóxico y citotóxico. Int. J. Morphol., 31(2):650-657, 2013.

RESUMEN: Los micronúcleos son fragmentos o cromosomas completos que quedan fuera del núcleo durante la mitosis; mediante su estudio se pueden evaluar los efectos de genotóxicos ambientales y ocupacionales. Esta prueba es ampliamente utilizada y es una alternativa eficaz, sencilla y económica para detectar la perdida de material genético. Por otra parte, la cavidad oral puede reflejar el estado de salud de los individuos, debido a que la mucosa que la recubre, puede presentar evidencias a nivel microscópico como macroscópico de cambios indicativos de enfermedad local sistémica o por exposición a sustancias tóxicas así como efectos secundarios por tratamientos. Dichas ventajas, favorecen su utilización en pruebas para evaluar genotóxicos o citotóxicos. La mucosa es una barrera protectora del resto del organismo, es un punto de contacto de agentes potencialmente peligrosos; por tanto, se torna susceptible de sufrir daños. El epitelio de revestimiento oral (60\%) es estratificado no queratinizado formado por células con abundante citoplasma, permite la penetración de colorantes y facilita la observación e identificación de características morfológicas del núcleo y membrana celular. Además la mucosa tiene elevada capacidad proliferativa y aunque esta particularidad mantiene la población celular constante, por otro lado, se vuelve más vulnerable a daño al ADN. Esto cobra relevancia ya que el $90 \%$ del cáncer tienen origen epitelial, así que la mucosa oral es usada para monitorear eventos genotóxicos tempranos causados por cancerígenos inhalados o ingeridos. Este epitelio es de fácil acceso, poco invasivo, por lo que al tomar la muestra a los individuos, se les genera mínimo estrés. Por todo lo anterior, el epitelio oral es un tejido ideal para aplicar la técnica de micronúcleos y detección de anormalidades nucleares sin necesidad de cultivos celulares, lo que representa una oportunidad para realizar estudios epidemiológicos en poblaciones de alto riesgo.

PALABRAS CLAVE: Micronúcleos; Mucosa oral; Anormalidades nucleares; Evento genotóxico y citotóxico.

\section{INTRODUCCIÓN}

Se ha descrito a la cavidad oral como el espejo que refleja la salud del individuo, pues en la mucosa que recubre la boca se observan cambios indicativos de enfermedad, además permite identificar efectos locales de tabaco o alcoholismo; puede revelar condiciones sistémicas, como la diabetes o la deficiencia vitamínica o bien, podría mostrar efectos secundarios originados por tratamientos quimioterapéuticos y radioterapia, ya que estas limitan la capacidad proliferativa de las células epiteliales, e incluso el tejido bucofaríngeo llega a adelgazarse al punto de formar ulceras (Squier \& Kremer, 2001). Las características del epitelio de la mucosa oral que favorecen su utilización en pruebas para evaluar genotóxicos o citotóxicos, es el punto de contacto que tiene con muchos agentes potencialmente peligrosos, por lo tanto; representa una barrera protectora para potenciales carcinógenos los que al ser metabolizados generarían múltiples metabolitos reactivos; este tejido sí protege al resto del organismo de que estos compuestos penetren a otros órganos; sin embargo, la mucosa al estar expuesta a estos agentes, es susceptible de sufrir daño. Por otra parte, es importante considerar que aproximadamente el $60 \%$ del total de la superficie de revestimiento oral es epitelio no queratinizado; lo que favorece la absorción de colorantes y facilita a la vez la observación e identificación de características morfológicas del núcleo y membrana celular a través del microscopio (Squier \& Kremer). Este epitelio, cuenta con una capacidad especial proliferativa, lo que permite que la población celular se mantenga constante; sin embargo,

\footnotetext{
* Profesor Investigador, Programa Internacional, Facultad de Medicina, Universidad Autónoma de Guadalajara. Zapopan, Jalisco, México.

${ }^{* *}$ Profesor Investigador Titular, Departamento. Salud pública, División de Ciencias Veterinarias, Centro Universitario de Ciencias Biológicas y Agropecuarias, Universidad de Guadalajara. Zapopan, Jalisco, México.
} 
esta característica lo vuelve más vulnerable a lesiones producidas en el ADN; por lo que cobra relevancia, ya que se calcula que el $90 \%$ de todos los tipos de cáncer tienen origen epitelial. Así que la mucosa oral podría ser usada para monitorear eventos genotóxicos tempranos causados por cancerígenos inhalados o ingeridos (Slack, 2000). Otra ventaja que presentan las células de este epitelio, es que presentan abundante citoplasma y conservan el núcleo al momento de ser exfoliadas (Squier \& Kremer); aunado a que son de fácil acceso y es posible colectarlas mediante técnicas mínimamente invasivas y relativamente indoloras, lo que produce mínimo estrés a las personas que se les toma la muestra por lo que es bien aceptada; e incluso, estas células se pueden estudiar sin necesidad de realizar cultivos celulares, facilitando y disminuyendo el costo de la técnica (Bonassi et al., 2011; Torres-Bugarín et al., 1998, 2009). Por lo tanto, es posible utilizar las células de la mucosa oral para evaluar el efecto genotóxico y citotóxico en poblaciones en alto riesgo, de forma sencilla, rápida y económica; esto mediante la prueba de micronúcleos $(\mathrm{MN})$ y detección de diversas anormalidades nucleares (AN) (Tolbert et al., 1992); por tanto, dicha técnica representa una oportunidad para realizar estudios epidemiológicos del impacto del estilo de vida, exposición ocupacional, nutrición, evolución de enfermedades crónico degenerativas, procesos de envejecimiento, exposición a genotóxicos, como: medicamentos, radioterapia, tabaco, alcohol, drogas, y por supuesto cáncer así como tratamientos antineoplásicos, entre otros (Bonassi et al.; Torres-Bugarín et al., 1998, 2004, 2007, 2009; Torres-Bugarín, 2000).

Células micronucleadas (CMN) en mucosa oral como parámetro de daño genotóxico. El monitoreo del daño genético en las poblaciones expuestas mediante la utilización de biomarcadores, se vislumbra como una herramienta útil en la prevención de tumores en la detección temprana de efectos secundarios, de enfermedades crónicodegenerativas y envejecimiento precoz (Bonassi et al.). Y los $\mathrm{MN}$ son una herramienta útil para ello, ya que se forman durante la transición de metafase-anafase en mitosis y pueden ser cromosomas completos rezagados ocasionado por un daño al uso mitótico (efecto aneuploidógeno) o bien, fragmentos de cromosomas sin centrómero (daño clastogénico); en ambos casos; no lograron incorporarse al núcleo de las células hijas (Schmid, 1975); lo que permite, diferenciarlos por el tamaño de los MN (Migliore et al., 1996) o la presencia del centrómero o cinetócoro (Afshari et al., 1989). Estos eventos pueden ocurrir espontáneamente, sin embargo, en presencia de ciertos agentes endógenos (Migliore et al.; Ramos-Remus et al., 2002; Rodríguez-Vázquez et al., 2000) o exógenos como se muestra en la Tabla I, la presencia de MN incrementan; convirtiéndose estos, en indicadores del efecto de agentes mutágenos, genotóxicos o teratógenos específicamente, micronucleogénicos (Heddle et al., 1991).

El conteo de los MN, es llevado a cabo fácilmente en cualquier tejido que se divida (Heddle et al.), como en el epitelio de mucosa oral (Bonassi et al.; Torres-Bugarín et al., 2004). Específicamente, los MN observados en células exfoliadas de tejido epitelial se forman en las células de la capa basal que es donde se lleva a cabo la división celular, esta migran a la superficie en el transcurso de 5 a 14 días, de tal manera que el monitoreo de poblaciones en este tejido puede reflejar el daño ocurrido durante este tiempo, la muestra se toma mediante un raspado de mucosa; se hace el extendido en portaobjetos perfectamente limpios, se fijan en etanol al $80 \%$, se tiñen con colorantes básicos o específicos para ADN (Holland et al., 2008; Nersesyan et al., 2006) y se analizan entre 500 a 4000 células (Ceppi et al., 2011), en las que se registran los MN encontrados (Fig. 1).

Otras anormalidades nucleares (AN) en células exfoliadas de mucosa oral. Además de los $\mathrm{MN}$ en células exfoliadas Tolbert et al. (1991), describen otras AN, las que además de ser fenómenos que podrían ocurrir en procesos normales de diferenciación celular, son indicadores de daño al ADN, citotoxicidad y muerte celular; ya que las alteraciones más sugestivas en la morfología de las células neoplásicas se producen en el núcleo, donde las modificaciones son en el tamaño, densidad y distribución de la cromatina; estas anormalidades se pueden distinguir de células normales por sus alteraciones ya sea en el citoplasma o en la morfología del núcleo, entre ellas se encuentran la cromatina condensada (CC), cariorrexis (CR), núcleo picnótico (NP), cariolisis (CL), núcleo lobulado también llamado prolongación nuclear, "bud cell" o "broken eggs" (NL, BE) y la presencia de células con dos núcleos, llamadas células binucleadas (BN). El mecanismo de formación o su significado biológico de cada una de estas AN no está muy bien esclarecido, sin embargo, bajo condiciones patológicas (obesidad, artritis

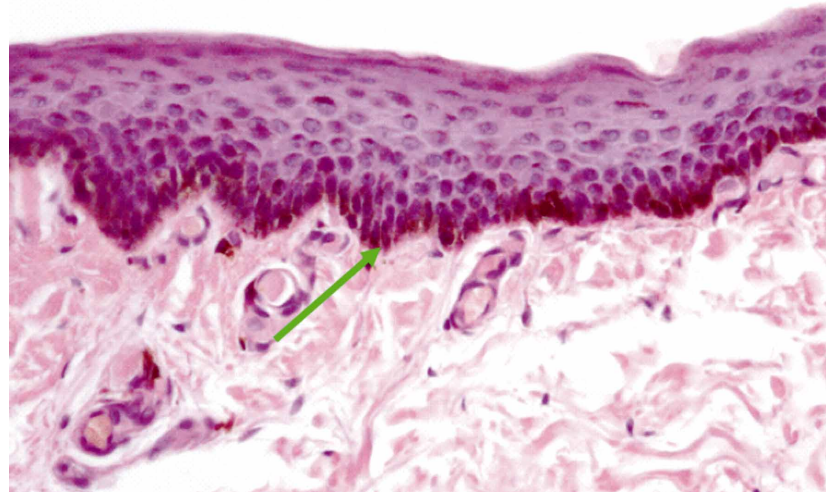

Fig. 1. Epitelio oral estratificado plano no queratinizado; En capa basal se lleva acabo la división celular, y es donde se originan los Micronúcleos (MN) y anormalidades nucleares (AN) para luego migrar a la superficie. 


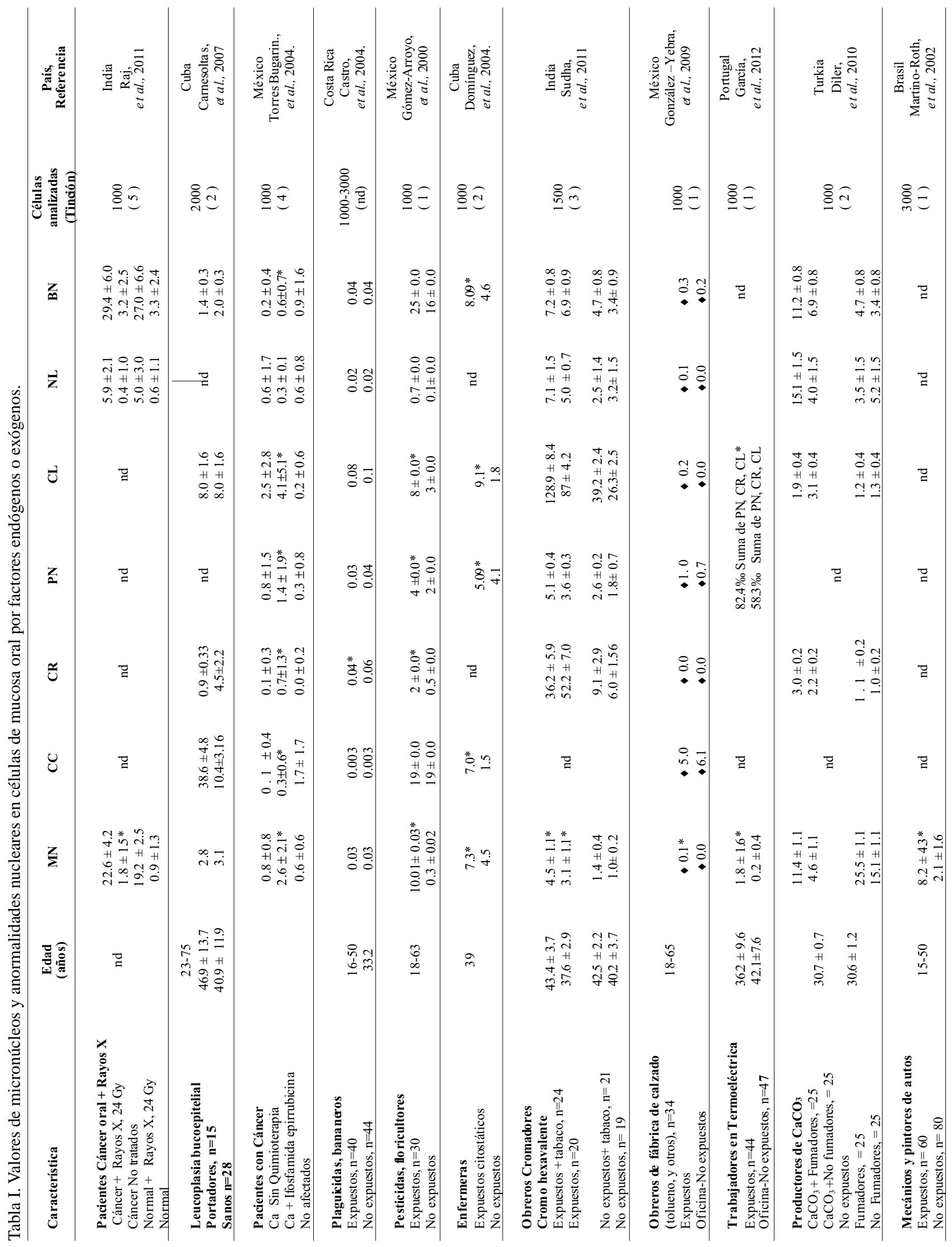


reumatoide, lupus eritematoso sistémico, diferentes tipos de cáncer, problemas hematológicos como: linfoma Inmunoblástico, Hodgkin; leucemia aguda megaloblástica, granulocítica crónica, linfocitica aguda; anemia linfocítica trombocitopénica; mieloma múltiple; entre otras) (Torres-Bugarin, 2000) o de exposición (tabaco, alcohol, drogas y quimioterapia antineoplásica); se observan altas frecuencias de células con AN (Tabla I). A este respecto, Raj et al., describen efecto dosis-respuesta, en la formación de MN y AN en pacientes con cáncer oral, por lo que se utiliza como un marcador de radiosensibilidad (Raj et al., 2011). Es importante mencionar, que también se observan en procesos de envejecimiento, como lo describen Thomas. (2008), en donde los MN, NL y las células BN están elevadas tanto en pacientes con síndrome de Down como en individuos de edades de 64 a 75 años. Por todas estas características ya señaladas, la prueba de identificación de AN se usa con frecuencia como marcador de daño al ADN (al analizar MN y NL); defectos en la citocinesis (por la presencia de varias células BN); evidencia de muerte celular (al observar CC, CR, PN, y CL); indicadores de diferentes etapas de necrosis (cuándo se presentan varias células con PN, CC, CR, CL); así como un identificador de respuesta al daño celular (al manifestarse células con PN y CC). Para su identificación, básicamente se usan los criterios establecidos por Tolbert en 1991 los cuales se describen a continuación (Thomas et al., 2008; Tolbert et al., 1991, 1992):

Células Normales $(\mathrm{CN})$ : el núcleo esta uniformemente teñido, es redondo u oval, se distinguen de las células basales porque son de mayor tamaño y el núcleo es más pequeño en relación al citoplasma. No contienen ningún otro cuerpo o estructura aparte del núcleo que contenga ADN, estas células son consideradas como células totalmente diferenciadas y no se observan divisiones celulares (Thomas et al., 2009; Tolbert et al., 1991) (Figs. 2a y 2b).

Célula Micronucleada (CMN): se caracteriza por la presencia de un núcleo principal y uno o más pequeñas estructuras nucleares denominadas $\mathrm{MN}$. Un $\mathrm{MN}$ tiene la forma redonda o almendrada y mide entre $1 / 3$ y 1/16 del núcleo principal, presenta la misma intensidad, textura y plano focal que el núcleo y es un fragmento o un cromosoma completo que al momento de la mitosis no se integra a uno de los núcleos de las células hijas (Thomas et al., 2009; Tolbert et al., 1991) (Fig. 2c).

Célula binucleada $(\mathrm{BN})$ : son células que contienen dos núcleos principales, usualmente los núcleos están muy próximos e incluso podrían hacer contacto, ambos con morfología y tinción similar a un núcleo normal. No parecen implicar una interacción directa con el ADN, sino que involucra la interferencia con los hechos ocurridos a finales de la división celular. Se piensa que es un evento que tiene lugar en dos etapas, en primer lugar ocurre la mitosis que dará origen a dos núcleos pero el citoplasma no se divide; en consecuencia, se forma una célula binucleada, misma que será eliminada si pertenece a un epitelio de revestimiento, pero si este fenómeno ocurre en una célula del epitelio basal o pertenece a otro 


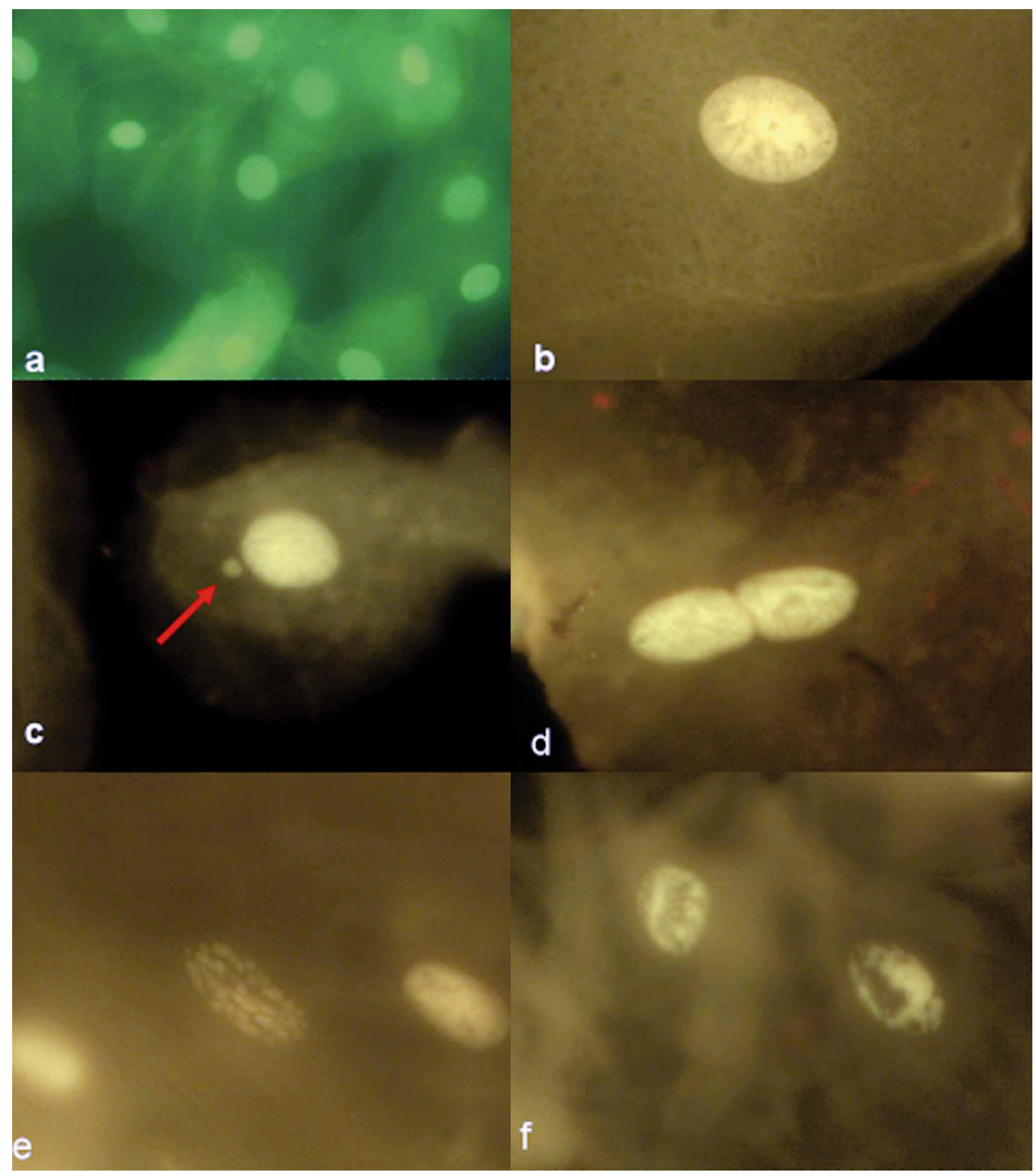

Fig. 2a. Células Normales, b núcleo normal, c. Célula micronúcleada (CMN); flecha micronúcleo (MN); d. Célula binucleada (BN), e. Cariorrexis (CR); f. Cromatina condensada (CC); Microscopio Binocular Mca. Carl Zeiss Mod Axiostar Plus.Fluorescencia IVFL Filtro de 450 a 490 Nanómetros, Objetivo Planacromático 100x/1.25 oil, cámara Axiocam Icc 1, Imágenes capturadas a 400 y a 1000 aumentos reales.

tejido entonces ambos núcleos de la célula binucleada entraran en mitosis al mismo tiempo, entonces cuando las membranas nucleares se desintegren, simultáneamente los cromosomas de ambos núcleos quedaran incluidos en el mismo huso y serán arrastrados juntos, así que en el momento de que la mitosis esté completa, habrá dos células 


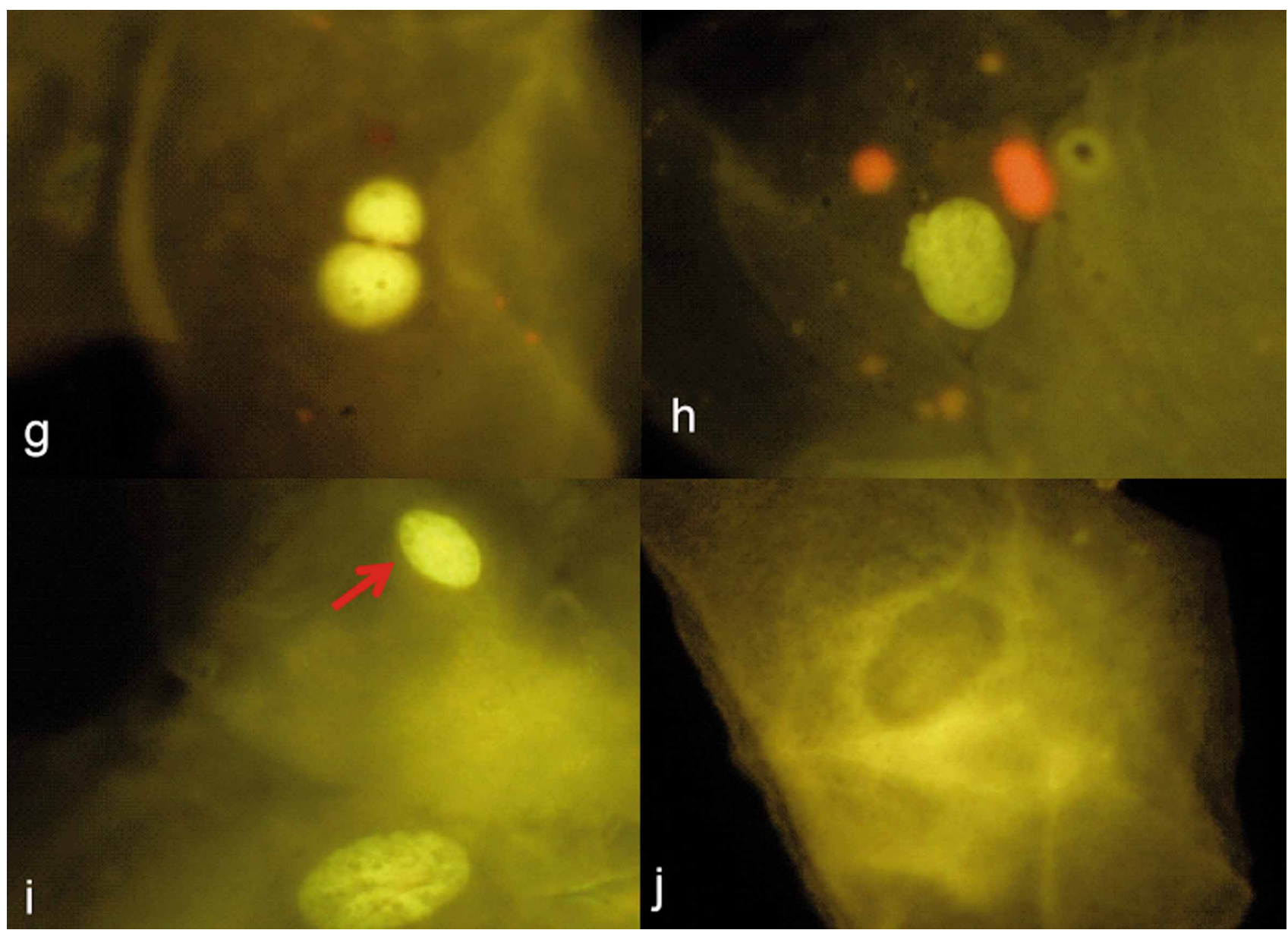

Fig. 2. g-h. Núcleo lobulado típico (NL); Núcleo lobulado, i. Núcleo picnotico, flecha (PN); j. Cariolisis (CL). Microscopio Binocular Mca. Carl Zeiss Mod Axiostar Plus. Fluorescencia IVFL Filtro de 450 a 490 Nanómetros, Objetivo Planacromático 100x/1.25 oil, cámara Axiocam Icc 1, Imágenes capturadas a 1000 aumentos reales.

con doble material genético cada una o bien, una célula tetrapaploide, si se repite el fenómeno de interrupción de la citocinesis (Shi \& \& King, 2005; Thomas et al., 2009; Tolbert et al., 1991) (Figs. 2d).

Cariorrexis (CR): células que presentan un núcleo que se caracteriza por agregación de la cromatina nuclear. Con respecto a las células de cromatina condensada, ellas tienen un patrón moteado nuclear indicativo de la fragmentación nuclear conducente a la eventual desintegración del núcleo. Estas células tal vez están pasando por una fase avanzada de apoptosis pero esto aún no ha sido comprobado (Thomas et al., 2009; Tolbert et al., 1991) (Fig. 2e).

Cromatina Condensada (CC): estas células tienen núcleos intensamente teñidos, con regiones condensadas o cromatina agregada exhibiendo un patrón nuclear moteado o estriado, es evidente que la cromatina está agregada en algunas regiones del núcleo; mientras que se pierde en otras áreas, así cuando la condensación es extensa da la apariencia de un núcleo fragmentado. estas células al igual que las células en carriorexis terminan con la fragmentación del núcleo, lo que conlleva la desintegración eventual y algunas veces aparecen como estructuras similares a los $\mathrm{MN}$, pero estas no deben de ser contabilizadaos como MN ya que su origen aun no es determinado, tal vez están en etapas tempranas de la apoptosis aunque dicha información aún no es concluyente (Thomas et al., 2009; Tolbert et al., 1991) (Fig. 2f).

\section{Núcleo lobulado o prolongación nuclear, broken} eggs (NL-BE): el núcleo presenta una constricción en un extremo, sugestivos de un proceso de eliminación de material nuclear por gemación. El lóbulo presenta las mismas características morfológicas y de tensión que el núcleo, pero el tamaño es de 1/3 a 1/4 del núcleo; Tolbert et al. (1991) los definió como "Broken egg", y Bhattathiri et al. (1998) describe estos fenómenos como "Nuclear buds" reconociéndolas como anormalidades nucleares. El origen y significado biológico de los BE en células exfoliadas aun no es totalmente comprendido y existen muy pocas publicaciones con- 
cernientes a este tema. Algunos investigadores consideran a los "nuclear buds" en linfocitos como indicadores de genotoxicidad, sin embargo, en células exfoliadas no es claro ya que frecuentemente en diversos procesos de salud y enfermedad aparecen con mayor frecuencia CMN en comparación a las "nuclear buds", por lo tanto; se puede asumir que las células con NL, no están asociadas a eventos genotóxicos, clastogénicos o aneuploidogénicos, pero tal vez sí a procesos degenerativos en la primera capa de células epiteliales (estrato germinativo) (Narsesyan, 2005; Thomas et al., 2009; Tolbert et al., 1991) (Figs. 2g y 2h).

Núcleo picnótico (PN): estas células se caracterizan por tener un núcleo pequeño, con alta densidad de material nuclear uniforme e intensamente teñido. El diámetro del nú- cleo es aproximadamente de 1/3 del núcleo normal y se piensa que estas células tal vez son una forma de muerte celular; sin embargo, el mecanismo preciso sigue siendo desconocido. Hasta el momento, solo se correlaciona con diferenciación y maduración de las células epiteliales (Thomas et al., 2009; Tolbert et al., 1991) (Fig. 2i).

Cariolisis (CL): estas células están completamente vacías de ADN y por lo tanto, no tienen núcleo. Es probable que representen una fase muy avanzada en el proceso de muerte celular. La correlación positiva entre células picnóticas y en cariolisis, sugiere que estas últimas, se derivan directamente a partir de células con cromatina condensada o indirectamente a través de células picnóticas (Thomas et al., 2009; Tolbert et al., 1991) (Fig. 2j).

TORRES-BUGARÍN, O. \& RAMOS-IBARRA, M. L. Utility micronucleus test and nuclear abnormalities in exfoliated cells of oral mucosa in the evaluation of genotoxic and cytotoxic damage. Int. J. Morphol., 31(2):650-657, 2013.

SUMMARY: Micronucleus are fragments or whole chromosomes that are outside the nucleus during mitosis. Through this study we can evaluate the environmental and occupational the genotoxic effects. This test is widely used because it is a very effective alternative, it is a simple, fast and inexpensive way to detect the loss of genetic material. Meanwhile a healthy oral cavity is evidenced because in the overlying mucosa changes indicative of local or systemic disease, toxic exposure and side effects of treatments can be observed. This favors their use in tests to assess the presence of genotoxins or cytotoxins. Although protective barrier from the rest of the body is the point of contact of potentially dangerous agents thus becoming susceptible to damage. Coating and oral epithelium (60\%) are formed by stratified non-keratinized cells with abundant cytoplasm, allowing the absorption of dyes and facilitating microscopic observation and identification of nucleus and membrane morphological characteristics. It has a particularly proliferative capacity, and even though this particularity maintains constant cell population, on the other hand, becomes more vulnerable to DNA damage. This information is relevant as $90 \%$ of all cancers are of epithelial origin. Therefore, the oral mucosa is used to monitor early events caused by inhaled or ingested genotoxic carcinogens. Epithelium is easily accessible and minimally invasive, thereby generating less stress when samples are obtained from study participants. In view of the above, oral epithelium tissue is ideal for implementing micronucleus assay and for the detection of nuclear abnormalities without the need for cell cultures, which presents a unique opportunity for epidemiological studies in high-risk populations.

KEY WORDS: Micronucleus; Oral mucosa; Nuclear abnormalities; Genotoxic y citotoxic event.

\section{REFERENCIAS BIBLIOGRÁFICAS}

Afshari, A. J.; McGregor, P. W.; Allen, J. W. \& Fuscoe, J. C. Centromere analysis of micronuclei induced by 2 aminoanthraquinone in cultured mouse splenocytes using both a gamma-satellite DNA probe and anti-kinetochore antibody. Environ. Mol. Mutagen., 24(2):96-102, 1994.

Bhattathiri, N. V.; Bindu, L.; Remani, P.; Chandralekha, B. \& Nair, K. M. Radiation-induced acute immediate nuclear abnormalities in oral cancer cells: serial cytologic evaluation. Acta Cytol., 42(5):1084-90, 1998.

Bonassi, S.; Coskun, E.; Ceppi, M.; Lando, C.; Bolognesi, C.; Burgaz, S.; et al. The HUman MicroNucleus project on eXfoLiated buccal cells (HUMN(XL)): the role of life-style, host factors, occupational exposures, health status, and assay protocol. Mutat. Res., 728(3):88-97, 2011.
Ceppi, M.; Gallo, F. \& Bonassi, S. Study design and statistical analysis of data in human population studies with the micronucleus assay. Mutagenesis, 26(1):247-52, 2011.

Heddle, J. A.; Cimino, M. C.; Hayashi, M.; Romagna, F.; Shelby, M. D.; Tucker, J. D.; et al. Micronuclei as an index of cytogenetic damage: past, present, and future. Environ. Mol. Mutagen., 18(4):277-91, 1991.

Holland, N.; Bolognesi, C.; Kirsch-Volders, M.; Bonassi, S.; Zeiger, E.; Knasmueller, S.; et al. The micronucleus assay in human buccal cells as a tool for biomonitoring DNA damage: the HUMN project perspective on current status and knowledge gaps. Mutat. Res., 659(1-2):93-108, 2008.

Migliore, L.; Cocchi, L. \& Scarpato, R. Detection of the centromere 
in micronuclei by fluorescence in situ hybridization: its application to the human lymphocyte micronucleus assay after treatment with four suspected aneugens. Mutagenesis, 11(3):285-90, 1996.

Nersesyan, A. K. Nuclear buds in exfoliated human cells. Mutat. Res., 588(1):64-8, 2005.

Nersesyan, A.; Kundi, M.; Atefie, K.; Schulte-Hermann, R. \& Knasmüller, S. Effect of staining procedures on the results of micronucleus assays with exfoliated oral mucosa cells. Cancer Epidemiol. Biomarkers Prev., 15(10):1835-40, 2006.

Ramos-Remus, C.; Dorazco-Barragan, G.; Aceves-Avila, F. J.; Alcaraz-Lopez, F.; Fuentes-Ramirez, F.; Michel-Diaz, J.; et al. Genotoxicity assessment using micronuclei assay in rheumatoid arthritis patients. Clin. Exp. Rheumatol., 20(2):20812,2002

Raj, V. \& Mahajan, S. Dose response relationship of nuclear changes with fractionated radiotherapy in assessing radiosensitivity of oral squamous cell carcinoma. J. Clin. Exp. Dent., 3(3):e193$200,2011$.

Rodríguez-Vázquez, M.; Sánchez-Ortiz, A.; Ramos-Remus, C.; Zúñiga, G. \& Torres-Bugarín, O. Evaluación de la genotoxicidad de la ciclofosfamida mediante prueba de micronúcleos en pacientes con lupus eritematoso sistémico. Rev. Mex. Reumatol., 15(2):41-5, 2000.

Schmid, W. The micronucleus test. Mutat. Res., 31(1):9-15, 1975.

Shi, Q. \& King, R. W. Chromosome nondisjunction yields tetraploid rather than aneuploid cells in human cell lines. Nature, 437(7061):1038-42, 2005.

Slack, J. M. Stem cells in epithelial tissues. Science, 287(5457):1431-3, 2000

Squier, C. A. \& Kremer, M. J. Biology of oral mucosa and esophagus. J. Natl. Cancer Inst. Monogr., (29):7-15, 2001.

Tolbert, P. E.; Shy, C. M. \& Allen, J. W. Micronuclei and other nuclear anomalies in buccal smears: a field test in snuff users. Am. J. Epidemiol., 134(8):840-50, 1991.

Tolbert, P. E.; Shy, C. M. \& Allen, J. W. Micronuclei and other nuclear anomalies in buccal smears: methods development. Mutat. Res., 271(1):69-77, 1992.

Thomas, P.; Harvey, S.; Gruner, T. \& Fenech, M. The buccal cytome and micronucleus frequency is substantially altered in Down's syndrome and normal ageing compared to young healthy controls. Mutat. Res., 638(1-2):37-47, 2008.

Thomas, P.; Holland, N.; Bolognesi, C.; Kirsch-Volders, M.; Bonassi, S.; Zeiger, E.; et al. Buccal micronucleus cytome assay. Nat Protoc., 4(6):825-37, 2009.
Torres-Bugarín, O.; Fernández-García A.; Torres-Mendoza, B. M.; Zavala-Aguirre, J. L.; Nava-Zavala, A. \& Zamora-Perez, A. L. Genetic profile of overweight and obese school-age children. Toxicol. Environ. Chem., 91(4):789-95, 2009.

Torres-Bugarín, O.; Covarrubias-Bugarín, R.; Zamora-Perez, A. L.; Torres-Mendoza, B. M.; García-Ulloa, M. \& MartínezSandoval, F. G. Anabolic androgenic steroids induce micronuclei in buccal mucosa cells of bodybuilders. $\mathrm{Br}$. $J$. Sports Med., 41(9):592-6, 2007.

Torres-Bugarín, O.; Ventura-Aguilar, A.; Zamora-Perez, A.; GómezMeda, B. C.; Ramos-Ibarra, M. L.; Morga-Villela, G.; et al. Evaluation of cisplatin $+5-\mathrm{FU}$, carboplatin $+5-\mathrm{FU}$, and ifosfamide + epirubicine regimens using the micronuclei test and nuclear abnormalities in the buccal mucosa. Mutat. Res., 565(1):91-101, 2004.

Torres-Bugarin, O. Evaluación de la genotoxicidad de las drogas antineoplásicas mediante el conteo de micronúcleos y otras anormalidades nucleares en mucosa bucal y micronúcleos en eritrocitos de sangre periférica. Tesis de Doctorado, Guadalajara, Universidad de Guadalajara, 2000.

Torres-Bugarín, O.; De Anda-Casillas, A.; Ramírez-Muñoz, M. P.; Sánchez-Corona, J.; Cantú, J. M. \& Zúñiga, G. Determination of diesel genotoxicity in firebreathers by micronuclei and nuclear abnormalities in buccal mucosa. Mutat. Res., 413(3):27781,1998

\author{
Dirección para Correspondencia: \\ Dra. en C. Olivia Torres-Bugarín \\ Profesor Investigador \\ Programa Internacional \\ Facultad de Medicina \\ Universidad Autónoma de Guadalajara \\ Zapopan \\ Jalisco \\ MÉXICO
}

Email: oliviatorres@hotmail.com

Recibido : 10-11-2012

Aceptado: 29-01-2013 